\title{
Diagnóstico de la mezcla de promoción de los programas de posgrado en las universidades públicas del estado Falcón
}

\author{
Marcano Aular, Yelitza* \\ Fernández, Gladys** \\ Pérez, Deisy***
}

\section{Resumen}

El presente estudio se enfocó en diagnosticar la mezcla de promoción en los Programas de Postgrado en las universidades públicas del Estado Falcón, abordando la caracterización del target group, la descripción de la mezcla de promoción y la caracterización de los canales personales e impersonales, justificánd ose en la necesidad de determinar la situación actual de la actividad de marketing que se realiza, para facilitar la labor gerencial de quienes la conducen. La investigación fue de tipo descriptiva, enmarcada dentro de la modalidad de proyecto factible. Se aplicó un censo poblacional a las unidades informantes, conformadas por los Coordinadores de los Programas de Postgrado, complementándose con la revisión de materiales promocionales. Como resultado de la caracterización del target group, se obtuvo que los encargados de realizar las actividades de promoción deben revisar la información que suministran a través de los diferentes canales personales e impersonales, a efectos de hacer que el mensaje sea mas efectivo. Además el estudio reveló que está claramente identificado el segmento del mercado y las características, comportamientos y necesidades de los individuos que solicitan cursar estudios de postgrado en las universidades públicas del estado Falcón. De igual manera, se determinó que es posible analizar y diseñar la mezcla de promoción del mercadeo educativo a través de la Venta personal, Publicidad, Promoción, Relaciones Públicas y los diferentes canales empleados para hacer llegar el mensaje. En cuanto al nivel de satisfacción de los encargados de dirigir y coordinar las actividades de promoción de los estudios de cuarto y quinto nivel, se considera que se satisface medianamente. Finalmente, se concluye que universidades deben

\section{Recibido: 09-06-08. Aceptado: 18-05-09}

* MSc. en Gerencia de Empresas. Profesora Asociada de la Universidad del Zulia, Núcleo Punto Fijo. Programa de Ciencia y Tecnología. E-mail: ymarcanoa@hotmail.com

** MSc. en Gerencia de Empresas. Profesora Asociada de la Universidad del Zulia, Núcleo Punto Fijo. Programa de Administración y Contaduría Pública. E-mail: hurtadoj@cantv.net

*** PostDoctora en Ciencias Gerenciales. Profesora Asociada de la Universidad del Zulia, Núcleo Punto Fijo. Programa de Administración y Contaduría Pública. E-mail: dperez@luz.ve 
aprovechar su valor de marca debido a que ésta representa el mayor valor institucional, al momento de promocionar sus productos educativos.

Palabras clave: Mercadeo educativo, Mezcla de Promoción, Universidades públicas, target group, Programas de Postgrado.

\title{
Diagnosis of the Promotional Mixture for Graduate Studies Programs at Public Universities in the State of Falcon
}

\begin{abstract}
This study focused on diagnosing the mixture for promoting graduate studies programs at public universities in the State of Falcon, emphasizing characterization of the target group, description of the promotional mixture and characterization of the personal and impersonal channels, justifying itself in the need to determine the current status of marketing activity carried out in order to facilitate the managerial work of those who direct it. The research was descriptive and framed in the feasible project mode. A population assessment was applied to the research participants, consisting of graduate program coordinators, complemented by a review of promotional material. As a result of the characterization of the target group, it was found that individuals in charge of performing promotional activities ought to check the information provided through the different personal and impersonal channels, in order to send a more effective message. Furthermore, the study revealed that the market segment and the characteristics, behaviours and needs of the individuals that apply for graduate courses in public universities of the State of Falcon are clearly identified. Likewise, it was determined that it is possible to analyze and design the mixture of educational market advertising through personal sales, publicity, promotion, public relations and the different channels used to spread the message. Regarding the satisfaction level of those in charge of directing and coordinating the promotional activities for fourth and fifth level studies, they were moderately satisfied. Finally, conclusions were that universities ought to take advantage of the value of their trademark, since it represents the highest institutional value when promoting their educational products.
\end{abstract}

Key words: Educational marketing, promotional mixture, public universities, target group, graduate studies programs.

\section{Introducción}

En Venezuela, los estudios de postgrado, representan el nivel educativo orientado a la formación especializada de los titulados universitarios, cuyo objetivo básico es elevar el nivel académico y profesional a través de la oferta de programas de especialización, maestría y doctorado, en la búsqueda de procesos de transformación e innovación reclamados por la sociedad, para lo cual es necesario contar con un perfil determinado generalmente por el tipo de estudios a realizar.

Cabe resaltar que en los últimos años, la educación superior se ha enfrentado a la aparición de nuevos competidores tanto del sector público como privado, lo cual amenaza con absorber el público objetivo, utilizando para ello satisfactores (productos/servicios) acordes a sus ne- 
cesidades, situación que puede influir en la adquisición o no de sus servicios.

Ante tal situación, puede inferirse que: la utilización inadecuada de una mezcla de promoción puede derivarse en pérdida de clientes, tanto actuales como futuros, igualmente un diseño del mensaje que no busque posicionarse en la mente del consumidor desembocaría en la disminución de imagen corporativa, de igual manera impediría tender puentes sólidos entre la institución y el mercado, con lo cual se imposibilitaría la oferta de productos complementarios, como pudiesen ser los resultados de las investigaciones derivadas de los estudios de especialización, maestría y doctorado. De igual manera, para aquellos clientes que no requieran de planes de formación a largo plazo, sería imposible desplazarlos hacia productos sustitutos que ofrecen las universidades, tales como los estudios de formación continua.

Sin duda alguna, la promoción es esencial para garantizar que los planes de acción, encaminados a establecer estrategias sólidas para posicionarse en el mercado y la mente del consumidor, sean realmente efectivos. Dada la dinámica actual del contexto donde se mueven las instituciones universitarias públicas, específicamente aquellas que ofertan Programas de Postgrado, conlleva a concurrir al mercado, en condiciones de competitividad con otras organizaciones similares; donde la calidad de los servicios ofrecidos, necesariamente deben responder a las expectativas de los consumidores.

Lo anteriormente expuesto permite inferir que, para enfrentar estos nuevos retos resulta imprescindible la proyección o promoción de las entidades universitarias públicas, con el objeto de adaptar la oferta educativa a los problemas actuales de la sociedad, teniendo en cuenta las necesidades de los clientes, para lo cual se debe hacer énfasis en el mercadeo educativo, por lo tanto, es necesario que las universidades comprendan la real utilidad de éste para ayudar a mejorar la gestión institucional y sobre todo sus mecanismos de promoción, entendiendo las limitaciones éticas para su uso.

Desde esta perspectiva, el mercadeo visto desde su dimensión integral, permitirá a los gerentes universitarios su aplicación en la administración de instituciones educativas, generando mayores beneficios, y posicionando la imagen corporativa de la institución, lo cual será un atributo, apreciado por los consumidores o clientes al momento de seleccionar una institución universitaria para realizar sus estudios de cuarto o quinto nivel. En este sentido, las razones que podrian diferenciar a los métodos utilizados por una u otra institución universitaria para la promoción de sus servicios, dependerá de las diferentes estructuras culturales de cada organización, de su historia y propósitos, además del nivel de conocimiento que los directivos o gerentes educativos tengan sobre el tema.

En este contexto, se plantea el presente estudio el cual tuvo como objetivo general: Diagnosticar la mezcla de promoción en los Programas de Postgrado de las universidades públicas del Estado Falcón y como objetivos específicos están los siguientes:

- Caracterizar el target group al cual van dirigidos los esfuerzos de promo- 
ción, en función del perfil establecido por los diferentes Programas de Postgrado de las Universidades públicas del Estado Falcón.

- Describir la mezcla de promoción que utilizan las universidades públicas del Estado Falcón, para estimular la demanda de sus Programas de Postgrado.

- Caracterizar los canales personales e impersonales de comunicación utilizados para la promoción de los Programas de Postgrado.

Con respecto a las unidades de estudio, cabe destacar que como requisitos necesarios y suficientes están: estar ubicadas en el Estado Falcón, ser universidades públicas e impartir Programas de Postgrado de cuarto y quinto nivel, conducentes a grado académico. Asimismo, es necesario acotar que para abordar la investigación se utilizó un censo poblacional, tomando en consideración que según Hurtado (2000:160) "no toda investigación requiere de un procedimiento de muestreo", en este caso en particular, el investigador tiene acceso directo a toda la población.

Entre las universidades objeto de estudio se destacan: la Universidad del Zulia, Universidad Nacional Experimental Francisco de Miranda, Universidad Nacional Experimental Politécnica de las Fuerzas Armadas, Universidad Nacional Abierta y Universidad Pedagógica Libertador, todas ubicadas en el Estado Falcón, conducentes a título de Especialistas, Magíster y Doctor.

Como unidades informantes se consideraron dieciocho (18) Directores y/o Coordinadores de Programas de
Postgrado de las universidades en estudio, pero considerando que existen coordinadores que administran más de un Programa de Postgrado, entonces, a efectos de la aplicación del instrumento se tomó como total, veintidós (22) fuentes. Igualmente, se realizó una revisión documental del material promocional de veinticinco (25) Programas de Postgrado, además se abordaron a los Delegados Docentes de Postgrado para obtener algunos datos específicos, con el fin de caracterizar el target group al cual van dirigidos los esfuerzos de promoción, en función del perfil establecido por los diferentes Programas de Postgrado en estudio.

Por otro lado, según el método empleado la investigación es de tipo descriptiva y de campo y la variable tratada; para este caso específico es la "Mezcla de Promoción", en el ámbito de las universidades públicas del Estado Falcón. El periodo de estudio estuvo comprendido entre Noviembre 2006 a Mayo 2007.

\section{La promoción en el marketing}

Examinando el papel de la promoción desde el punto de vista del marketing, se puede decir que ésta sirve para lograr los objetivos de una organización. En ella "se usan diversas herramientas para tres funciones promociónales indispensables: informar, persuadir y comunicar un recordatorio al auditorio meta" (Stanton et al., 2004: 567). El autor expresa la importancia relativa de esas funciones dependiendo de las circunstancias que enfrente la organización; con respecto a la función de informar se debe lograr que el mercado meta entienda cuáles son 
Ios beneficios proporcionados por el producto o servicio, cómo funciona y cómo obtenerlo; de igual manera, el objetivo de la persuasión ante un mercado donde la competencia es intensa, debe ser un elemento a considerar y por último la comunicación es importante para recordarle a los consumidores la disponibilidad de un producto y su potencial para satisfacer.

Es importante para las empresas, entender la diversidad de la demanda de los consumidores cuando varían sus ofertas de servicios para atraer a diferentes segmentos; por otro lado, los vendedores de servicios se enfrentan a varios problemas únicos en el posicionamiento y en la promoción de sus ofertas:

Primero, debido a que los servicios son intangibles, es más difícil crear una imagen de servicios, el riesgo percibido será más alto y esto se verá aumentado por la probabilidad de variabilidad en la entrega de servicios de una ocasión a la siguiente. Segundo, debido a que los servicios son perecederos, la promoción se vuelve particularmente importante. Tercero, debido a que los servicios se producen y se consumen en forma simultánea, los consumidores tienden a identificar el servicio con los proveedores del mismo, por lo que es crítico que estas organizaciones maximicen la comprensión de los empleados y la orientación hacia los consumidores en cada etapa del servicio para asegurar la satisfacción del cliente.

Debido a la intangibilidad de los servicios, la imagen se vuelve un factor fundamental para diferenciarlo de su competencia, aquí el objetivo del marketing es capacitar al consumidor para que articule una imagen específica con un nombre de marca específica; por otra parte, los servicios son variables y las empresas están en posición de ofrecer varias versiones a diferentes segmentos de mercado.

El diseño del medio ambiente de los servicios es un aspecto importante de la estrategia de comercialización, e influye agudamente sobre las impresiones $y$ el comportamiento del consumidor.

Entre los principios fundamentales de la promoción están: (a) Utilizar mensajes claros sin ambigüedades, (b) Destacar los beneficios de los servicios, (c) Solo prometer lo que se puede dar, (d) Proporcionar Publicidad para los empleados, (e) Obtener y mantener la colaboración de los clientes en el proceso de producción del servicio, (f) Crear comunicación verbal entre las partes involucradas, (g) Dar pistas tangibles (símbolos, temas, formatos), (h) Dar continuidad a la publicidad y (i) Eliminar la ansiedad después de la compra.

\subsection{La mezcla de promoción y sus métodos}

La mezcla de promoción desde el punto de vista teórico es la "combinación de ventas personales, publicidad, promoción de ventas y relaciones públicas de una organización" (Stanton et al., 2004: 577). En tal sentido, es necesario considerar la diferenciación del producto, el posicionamiento, la segmentación de mercado, el comercio y el manejo de marca, realizando esfuerzos significativos para lograr una promoción eficaz. Continúa acotando el autor, que el diseño de una mezcla promocional efectiva comprende un número de decisiones estratégicas en torno a cinco (5) factores: 
- Auditorio meta: elemento que influye definitivamente en las decisiones sobre la mezcla promocional. Su objetivo puede ser los clientes actuales, los nuevos prospectos y los intermediarios.

- Objetivo del esfuerzo de promoción: la meta de la promoción es poner al prospecto al final o en la etapa de compra, pero en la mayoría de los casos esto no es posible mientras la persona no haya pasado por las etapas iniciales, es decir crear conocimiento de las ventajas de un producto.

- Naturaleza del producto: Existen algunos atributos de los productos que influyen en la mezcla de promoción, tales como el valor unitario del producto o servicio, el grado de individualización y el servicio preventa y posventa.

- La cantidad de dinero disponible.

- Etapa en el ciclo de vida del producto: cada etapa del ciclo de vida de un producto influye en las estrategias de promoción del mismo.

Kotler (2000), Schoell y Guiltinan (1991), plantean que para promocionar un producto o servicio existen cuatro formas o métodos: la venta personal, la publicidad, la promoción de ventas y las relaciones públicas. A continuación se describen cada una de ellas, en función de la visión de Kotler: (a) La venta personal, consiste en la presentación directa de un producto a un cliente prospecto por un representante de la organización que lo vende. Estas se efectúan cara a cara o por teléfono y pueden dirigirse a una persona de negocios o a un consumidor final. (b) La publicidad por su parte es una co- municación no personal, generalmente pagada por un patrocinador claramente identificado, que promueve ideas, organizaciones o productos. Suelen transmitirse por televisión, radio, Internet u otros medios impresos (diarios y revistas). (c) La promoción de ventas, es ideada para completar la publicidad y facilitar las ventas personales. Consiste básicamente en ofrecer un incentivo temporal para alentar a una venta o una compra y comprende un amplio espectro de actividades, como patrocinios de eventos, concursos, exposiciones comerciales o industriales, exhibiciones en tiendas, reembolsos, muestras, premios, descuentos y cupones. (d) Por último, las relaciones públicas abarcan una gran variedad de esfuerzos de comunicación para contribuir a actitudes y opiniones generalmente favorables hacia una organización y sus productos. Esta no incluye un mensaje de ventas específico y sus objetivos pueden ser: organizaciones gubernamentales y grupos de interés especial, entre otros. Una forma especial de las relaciones públicas es la publicidad no pagada, donde la organización que es tema de la publicidad tiene poco o ningún control sobre ella; ya que aparece como noticia y en consecuencia le da mayor credibilidad.

\subsection{La mezcla de marketing en las organizaciones educativas}

Rodríguez y García (2002), definen el marketing educativo como el proceso de investigación de las necesidades sociales para desarrollar servicios educativos tendientes a satisfacerlas, acorde a su valor percibido, distribuido en tiempo y lugar, y éticamente promocionados para 
generar bienestar entre individuos y organizaciones. Continúa acotando el autor, la existencia de tres tipos de aproximaciones en el ejercicio del Marketing: Un marketing mínimo (entendido como aquel que se aplica a los servicios profesionales, sujeto a los códigos de éticas de sus colegiaturas, donde los grados de libertad para promocionarse son escasos e indirectos), un marketing equilibrado (aplicable a instituciones educativas, organizaciones de salud y entidades sin fines de lucro, donde las acciones promociónales deben alejarse de un sentido mercantilista que es sancionado por la sociedad) y un marketing comercial (el cual desde su propia acción mercantilista pretende influir en las decisiones de los consumidores).

En el caso de las decisiones de marketing en las instituciones educativas, existe un conjunto de variables controlables como: desarrollo de las propuestas pedagógicas curriculares, servicios extra-programáticos, justo precio, la distribución del servicio en diferentes sedes, la evaluación de los procesos de aprendizaje enseñanza, gestión de las instalaciones y el equipamiento. El correcto manejo de éstas debe orientar la actividad de marketing en las instituciones educativas al mejoramiento de la calidad de los servicios.

Todos los esfuerzos comerciales de una organización convergen hacia un punto, determinar la mezcla de promoción adecuada, entendida ésta como "el conjunto de herramientas de marketing a utilizar por las empresas para conseguir sus objetivos comerciales en relación con su público objetivo" (Kotler, 2000). En el mercadeo educacional la mezcla del marketing tiene ciertas particularidades que van desde cambios en la denominación de lo que se conoce como las cuatro (4) "P" (Producto, Precio, Plaza y Promoción).

a) El satisfactor de la Educación Superior (producto)

En la mercadotecnia tradicional las organizaciones deben estudiar las necesidades y/o deseos del grupo de clientes al cual esperan llegar. En la mercadotecnia educativa se debe hacer de igual modo un estudio de las necesidades y problemas de la demanda. Por tanto, se diseña el Satisfactor de manera que se retribuya a los usuarios con un producto $o$ servicio con un valor agregado, mejor que el ofrecido por la competencia. Por otra parte, es necesario aclarar que en el diseño del producto educativo (satisfactor), se debe tener en cuenta el caso específico de la docencia; en ella la oferta está condicionada por los planes de estudios nacionales, de ahí que se debe llegar a un equilibrio a la hora de diseñar el satisfactor entre la oferta y la demanda del mismo.

El diseño del satisfactor en la educación superior es algo de gran complejidad, pues entraña una decisión la cual repercutirá en el futuro de un individuo. En gran medida la complejidad relativa al producto del mercado educativo está influenciada por la motivación de la compra, el estudiante cuando decide ingresar en una institución universitaria no busca propiamente el producto en sí, sino una oportunidad de mejorar el futuro, la compra es la posibilidad de obtener un empleo de mayor remuneración.

Con respecto a este caso, muchos economistas estudiosos de la economía de la educación plantean que el estudio 
de la demanda educacional es más atractivo que el estudio de la oferta, la misma es derivada de la demanda de empleo, se puede decir entonces que lo que realmente se compra de una Universidad en términos de demandante potencial, es un futuro determinado.

\section{b) El precio (el intercambio).}

Una oferta educativa no sólo es atractiva y aceptada por los clientes por la capacidad que tiene el producto de satisfacer las necesidades, sino también depende de su precio. Las instituciones adscritas al Estado, colegios, universidades, e institutos de diversa índole, no tienen ánimo de lucro. El precio de la educación es esencialmente un intercambio entre universidad y usuario, los diferentes programas educativos deben estimar los precios monetarios y los precios no monetarios que permitan el acceso a los interesados en adquirir o utilizar los servicios educativos.

\section{c) La distribución del producto educativo (la facilitación)}

Stern y El- Ansary (1988) definen los canales de distribución como una red de instituciones y de organismos involucrados en la tarea de desplazar productos desde los puntos de producción hasta los puntos de consumo. Desde el punto de vista del mercadeo educativo la (facilitación) distribución es una de las variables de mayor dificultad, a la hora de adaptarla; infiriendo en lo anteriormente planteado, las universidades vistas como empresas que ofrecen satisfactores, tienen que crear los mecanismos para atraer a los "clientes", ofreciendo facilidades que no estén restringidas por la ubicación geográfica. Por su parte, la facilitación en la educación debe prestar aten- ción a la proliferación que han tenido los cursos de educación universitaria a distancia en países como México y España, entre otros, superando las barreras geográficas y de tiempo.

De esta manera, el rol de la distribución de un producto educativo es facilitar el acceso del mismo a los interesados, entonces su objetivo es brindarle a los usuarios toda la información que les permita dar respuesta a las siguientes preguntas ¿dónde ir?, ¿cuándo ir? y ¿cómo actuar?

d) La promoción de la oferta educativa (la comunicación)

Una acertada estrategia de mercadotecnia para diferentes programas y servicios educativos, necesita informar a su mercado meta acerca de sus objetivos, programas y actividades para que estos se sientan interesados por la organización. Por tanto, la comunicación tiene la tarea fundamental de conseguir que el producto educativo se vuelva aceptable y deseable para los interesados (audiencia) que constituyen el grupo meta. Esta estrategia en la mercadotecnia tradicional se conoce como promoción y en la mercadotecnia educativa se denomina comunicación.

Se infiere, en referencia a la comunicación en el mercadeo educativo, que los esfuerzos en este sentido deben de ir dirigidos esencialmente a su público objetivo, para lograrlo es necesario: (a) Identificar los problemas o requerimientos del grupo destinatario de interés; (b) Diseñar mensajes que aborden los obstáculos y comuniquen al mercado todo lo que se deba conocer respecto al producto; (c) Probar los mensajes en una muestra del grupo de destinatarios objeti- 
vo; (d) Producir y difundir los mensajes para los medios adecuados y; (e) Evaluar la efectividad de la campaña de comunicación.

\section{Resultados}

A continuación se presentan los resultados derivados del estudio de la variable de investigación: mezcla de promoción, la cual consiste en una combinación específica de instrumentos de publicidad, venta personal, promoción de ventas y relaciones públicas, que la organización utiliza para lograr sus objetivos de publicidad y de mercadotecnia (Kotler, 2000), donde cada elemento tiene características definidas que determinan la función que puede representar en un programa de promoción, además se presentan los resultados relacionados con la caracterización del target group al cual van dirigidos los esfuerzos de promoción. Cabe destacar, que el análisis presentado a continuación está en función de las veintidós (22) fuentes consultadas.

\subsection{Caracterización del target group}

Los profesionales universitarios constituyen un público meta muy diverso conformado por médicos, abogados, ingenieros, administradores, o cualquier otro que haya recibido una certificación o título a nivel universitario. Este público según refiere Stanton et al. (2004), exige una clasificación aparte porque sus integrantes tienen necesidades e intereses particulares. Es por ello, que la publicidad dirigida a los profesionales se ocupa de productos y servicios (satisfactores) que con frecuencia se diseñan de manera ex- presa, para servir a necesidades específicas. Además, el lenguaje y las imágenes de la publicidad para este público meta suelen contener una terminología esotérica y circunstancias peculiares, que los integrantes de las profesiones reconocen con facilidad.

A efecto de esta investigación, para la determinación del target group, una vez realizada la recopilación y revisión de la información disponible en trípticos, folletos, páginas web y avisos de prensa, a veinticinco (25) Programas de Postgrado, estos resultados organizados en cuadros, ordenados e interpretados para obtener los datos requeridos. A efecto de caracterizar el target group al cual van dirigidos los esfuerzos de promoción, en función del perfil establecido por los diferentes Programas de Postgrado de las Universidades públicas del Estado Falcón, se consideraron los siguientes indicadores: Tipo de Programa (Especialización, Maestría, Doctorado), Título profesional solicitado, Trabajo en el área, Componente Personal, Habilidades y/o Destrezas, y Líneas de Investigación.

Al hacer referencia a los conocimientos, se considera el título de pregrado solicitado para cursar los estudios de postgrado, se incluyen otros indicadores tales como "Tipo de Programa de Postgrado", además de los parámetros "Componente personal", "Trabajo en el área" y "Habilidades previas" que debe tener el "comprador" para adquirir el producto educativo, o para ser seleccionado.

Como resultado del análisis se obtuvo que los materiales empleados por las unidades informantes para promocionar sus Programas de Postgrado, tienen las siguientes características: 
- De los 25 Programas de Postgrado analizados, en un $85 \%$, está claramente identificado el perfil profesional (Título de pregrado solicitado); por otra parte, el hecho de no indicar el título profesional solicitado puede causar ruido en el mensaje y afectar la decisión del aspirante, aun más si el programa de postgrado es de tipo multidisciplinario.

- Por otro lado, en un $58 \%$ de los Programas de Postgrado en estudio, no está especificado si el solicitante debe "Trabajar en el área", a la que pertenece el postgrado ofertado y en un $12 \%$ este aspecto no es una condición en extremo necesaria, en tanto que en un $30 \%$ si lo exige.

- Con respecto al indicador "Componente personal", sólo un $19 \%$ del total de 25 Programas de Postgrado estudiados, especifica cuales son los atributos que debería tener el solicitante, en función de los requerimientos del Programa de Postgrado que aspira a cursar. De indicarlo, sería equivalente a suministrar información también sobre el producto o satisfactor en si. Algunas de las condiciones requeridas, según la revisión efectuada son: Capacidad de liderazgo, Actitud para el estudio, y capacidad para trabajar en equipos multidisciplinarios.

- Las "Habilidades previas" del participante, en un $35 \%$ del total, es indicado en los materiales promocionales. Como se puede apreciar, el porcentaje es bajo, si se considera que proporcionar información al aspirante a cursar un programa de postgrado, sobre cuales son las habilidades o destre- zas para hacer mas eficiente su permanencia en el mismo; sin duda contribuiría a que la decisión de compra o adquisición del satisfactor esté fundamentada en base a los criterios solicitados. Los aspectos solicitados en general son: la experticia en el área, manejo instrumental de otros idiomas diferentes al español y la capacidad para conducir aprendizaje científico.

- Cabe destacar, que el factor investigación, es un elemento primordial para los estudios de cuarto y quinto nivel, por tanto, se debería resaltar dentro del mensaje a transmitir; pero según la revisión efectuada se aprecia que prácticamente no se hace énfasis en éste; aún cuando se considera como un elemento clave de estos estudios. Sólo $31 \%$ de los materiales revisados indica cuales son las líneas de investigación disponibles para el Programa de Postgrado ofertado; el desconocimiento de cuáles son las directrices que deben guiar el proceso de investigación durante la escolaridad y su posterior presentación y defensa del trabajo especial de grado, tesis de grado o tesis doctoral, según sea el caso, se considera desfavorable porque en muchos casos no contribuye al logro de la obtención del título de postgrado.

- Con respecto al indicador "Tipo de Programa de Postgrado", en un 100\%, se indica el tipo de programa, pero no queda claro para el lector en qué se diferencia un programa de postgrado conducente a grado de especialista, magíster o de doctor; aún cuando en algunos casos esto pareciera estar tá- 
citamente indicado en los objetivos específicos del programa.

En consecuencia, los encargados de realizar las actividades de promoción de los Programas de Postgrado en las universidades, deben revisar cual es la información que actualmente proporcionan al diseñar los diferentes canales impersonales que utilizan para promocionar sus ofertas de estudio de cuarto y quinto nivel, en pro de hacer más eficiente la difusión del mensaje y la consiguiente persuasión del público meta.

\subsection{Mezcla de promoción}

Un elemento decisivo del éxito de una organización es la capacidad de segmentar adecuadamente su mercado (Stanton et al., 2004). Según los resultados obtenidos de las 22 unidades informantes consultadas, se evidencia que un $91 \%$ del total tiene claramente identificado cual es su segmento del mercado, lo cual significa que las características, comportamientos y necesidades de los individuos que solicitan cursar estudios de postgrado, son consideradas al momento de ofertar dichos programas.

En ese sentido y considerando los planteamientos de Kotler (2000) se puede adoptar un enfoque de mercadotecnia orientada al mercado meta; debido a que este permitiría aun más resaltar la identificación de segmentos del mercado, selección de uno o más de ellos y el desarrollo de mezclas de productos y/o servicios de mercadotecnia, ajustados a cada uno de ellos.

\section{a) Fuerza de ventas}

Considerando la posición de Stanton et al. (2004), el proceso de venta personal que realiza la fuerza de ventas, consta de cuatro pasos, a saber, los dos primeros son la prospección de los compradores potenciales y el acercamiento preliminar a cada uno de ellos. El tercer paso es la presentación de la venta, que incluye despertar el deseo e interés del comprador, contestar las objeciones y cerrar la venta, por último está la actividad de seguimiento o servicio postventa.

Una vez procesados los datos recopilados del cuestionario, con respecto al uso de la fuerza de ventas para promover la participación de los profesionales en los Programas de Postgrado ofertados por la universidad, se encontró que un $43 \%$ de los encuestados, utiliza personal para la promoción directa del servicio ofrecido. Sin embargo, es importante destacar que de hacerlo se podría impulsar aun más el conocimiento a los clientes potenciales sobre los Programas de Postgrado proporcionados. De igual manera, permitiría consolidar la lealtad de los clientes, resaltando la necesidad de establecer vínculos entre la universidad y los clientes.

Es necesario entonces, que el personal que forme parte de la fuerza de ventas, cuente con las siguientes características: sea personal ordinario (preferiblemente docentes con estudios de postgrado en el área ofertada), estar informado de las situaciones que forman el entorno de la universidad, precios, competencia, así como de las necesidades y actitudes de los clientes potenciales frente al servicio ofrecido. Así como también se considera importante, que tanto la fuerza de ventas de gran experiencia como los nuevos prospectos, necesitan un buen programa de capacitación para mejorar sus habilidades de venta, conocer mejor los 
productos y perfeccionar las prácticas gerenciales de su tiempo y territorio (Stanton et al., 2004).

\section{b) Materiales promocionales}

Sin duda alguna, en la promoción de cualquier servicio o producto, es muy importante el uso de materiales como folletos, avisos, trípticos, carteleras informativas, CD's tutoriales, material POP (Point of Parchase o Punto de Venta), entre otros, para atraer la atención de los clientes, en esto concuerdan autores como Russell et al. (2006) y Stanton et al. (2004). Coincidiendo con los encuestados, quienes en un $91 \%$ emplean materiales promocionales para apoyar la promoción de los Programas de Postgrado.

Con este tipo de materiales, por lo general de bajo costo, se puede incorporar información útil para los clientes, tal como: dirección, teléfonos, URL y correo-e, oferta de Programas de Postgrado y sus líneas de investigación, además de resaltar el nombre de la universidad. Se concluye este punto enfatizando un pensamiento de O'Guinn et al. (1998; 381 ), cuando resaltan que "no importa cuan grande sea un plan de mercadotecnia y no importa cuan talentosos o visionarios sean los estrategas publicitarios, una mala colocación del mensaje afectará incluso los mejores planes"; esto haciendo alusión al empleo adecuado de los materiales promocionales.

\section{c) Recursos financieros}

Un $73 \%$ de los encuestados manifiesta que si existen limitaciones en cuanto a los recursos financieros para invertir en la promoción de los Programas de Postgrado. Aún más, si se considera que establecer los presupuestos de promoción es una tarea en extremo desafiante porque la administración no cuenta con normas o estándares confiables para determinar cuánto gastar en publicidad, ventas personales y el resto de la mezcla de promoción o que tanto del presupuesto total se ha de asignar a cada componente de la mezcla (Stanton et al., 2004).

d) Participación de profesiona-

\section{les del área de la mercadotecnia}

La función de los expertos y profesionales del marketing es influir en el comportamiento de los clientes. Para llevarlo a cabo cuentan con varias herramientas en términos generales, entre las cuales destacan: el diseño de un producto o servicio, el precio al cual se ofrece, el mensaje con el cual se describe y el lugar donde está disponible (Stanton et al.; 2004). Un $100 \%$ de los encuestados manifiesta que la decisión sobre la mezcla adecuada de mercadeo, no está apoyada en la participación de profesionales del área de mercadotecnia, situación que induce a pensar que actividades como las señaladas por Stanton et al., no se estarían ejecutando o pudiese estar signada la labor por acciones no muy eficaces. nales

e) Uso de estrategias promocio-

Por otro lado, un $59 \%$ declara que si diseña estrategias promocionales para motivar a su segmento del mercado y así influir en la selección de sus productos educativos o satisfactores. Sobre este particular, O'Guinn et al. (1998), destacan que los objetivos de medios promocionales constituyen la base para la selección de éstos y el verdadero poder de un plan de medios está en su estrategia, es decir, aquellas decisiones tomadas respecto al alcance y frecuencia de un vehículo de medios (entiéndase como la 
opción específica para la colocación de una clase de medios), la continuidad de su colocación en los mismos, la duplicación del público y la duración y tamaño de los anuncios. Las decisiones del estratega de medios ayuda a asegurar que los mensajes colocados en ellos tengan tanta influencia como sea posible.

\section{f) Apoyo de aplicaciones infor- máticas}

Es necesario hacer notar que aun cuando los encuestados manifestaron en un porcentaje elevado, $82 \%$, el uso de aplicaciones informáticas para dar soporte a la toma de decisiones promocionales; se pudo detectar en función de la observación directa y las entrevistas realizadas, que ninguno de ellos cuenta con un sistema de información diseñado para tal fin, que en verdad les permita generar, analizar, difundir, almacenar y recuperar la información para utilizarla en la toma de decisiones de marketing, sólo se basan en aplicaciones ofimáticas, las cuales en el mejor de los casos le proporcionan estadísticas y visualización sobre el comportamiento de los participantes; información manejada principalmente por las secretarías docentes de postgrado o direcciones docentes del mismo.

Según Stanton et al. (2004) un sistema de información ideal debería: generar informes periódicos y estudios recurrentes según se vayan necesitando, integrar datos nuevos y viejos para actualizar la información e identificar las tendencias, y analizar los datos aplicando modelos matemáticos que representan el mundo real.

\section{g) Marca}

A efectos de este estudio se consideró como marca, el nombre y el prestigio de la universidad que ofrece los Progra- mas de Postgrado. Tal como acota Cobra (2001), la marca se construye en el marco mental de las personas, sin embargo, no siempre simboliza lo que los consumidores decodifican de su nombre y aunque una empresa, o institución sea muy competente, o destacada en el mercado, su marca no puede emplearse de manera indefinida, ni mucho menos pensar que no habrá necesidad de reposicionarla; la investigación por su parte reveló en un $68 \%$ el aprovechamiento del nombre o reputación de la universidad para destacar sus mensajes de promoción.

Un 100\% manifiesta que el prestigio de la Universidad influye al momento de realizar la promoción de sus Programas de Postgrado.

\section{h) Penetración del mensaje}

Aun cuando un $77 \%$ considera que el diseño del mensaje para la promoción, está en consonancia con el target al cual va dirigido, se encontró en función de la revisión documental realizada, que no existe un mensaje como tal. Es decir, el mensaje entendido como la suma de signos y señales que intentan transmitir una o más ideas, como slogan o frases cortas que resuman el mensaje, no está presente en el material revisado. A tal efecto, Coughlan et al. (2006) plantean que el diseño del mensaje publicitario es un proceso creativo y debe decir qué se ofrece y por qué puede interesarle al destinatario del mensaje, a lo cual añaden Schoell y Guiltinan (1991) que también se pueden incluir experiencias.

En el caso de la oferta de los Programas de Postgrado, el mensaje podría relacionarse por los objetivos del postgrado, y la justificación del mismo; si se consideran estos como los mensajes, enton- 
ces se puede acotar que todos los instrumentos para la promoción revisados cuentan con él; haciendo énfasis en las características específicas de la población-objetivo a la cual van destinados.

A su vez cuando se preguntó sobre lo que se busca al transmitir el mensaje promocional, se obtuvo lo siguiente: Coinciden en un $91 \%$ que se persigue: atender a las necesidades de los clientes potenciales y proveer información acerca de la oferta educativa al mercado potencial y atraer estudiantes para así estimular sus inscripciones; en un $73 \%$ se busca mejorar la imagen de los Programas de Postgrado ofrecidos por la universidad y en igual porcentaje crear la imagen de los Programas de Postgrado ofertados. De igual manera, persiguen mantener la imagen de los mismos, en un $68 \%$, a su vez construir lealtad en los egresados de postgrado, representa sólo el 46\%. Tal como se puede apreciar en su mayor porcentaje, el mensaje hace énfasis en los beneficios que traería el satisfactor (producto educativo) al público objetivo, suministrando para ello la información necesaria.

Con respecto a si utilizan mecanismos para obtener información sobre la penetración del mensaje de promoción, un $73 \%$ revela que no. Situación que imposibilita el poder contar con un feedback sobre lo que piensa el mercado objetivo, escenario que pudiese mejorarse al emplear encuestas rápidas, al mercado ya cautivo, aplicadas vía correo electrónico o directamente en las instalaciones donde se presta el servicio. Así pues, tan importante es el diseño y difusión del mensaje como evaluar o monitorear la penetración del mismo.

\subsection{Canales de comunicación personales e impersonales}

\section{a) Eventos}

Una oportunidad para promocionar los Programas de Postgrado, lo constituyen la utilización de canales personales, por ejemplo, la participación en eventos especiales, como pueden ser Jornadas de Investigación y Postgrado, Congresos, Ferias Científicas, Aniversarios de la universidad, e incluso eventos organizados en alianzas con otras instituciones universitarias, empresas públicas o privadas, en pro de exhibir o mostrar la oferta académica de sus estudios de cuarto y quinto nivel.

Sin embargo, según los resultados del cuestionario un $45 \%$ no utiliza este tipo de eventos para promover sus Programas de Postgrado; desaprovechando la oportunidad y beneficios que ello significa.

Algunas de las ventajas que aporta por ejemplo, la participación en ferias, según García (2007), serían: presentar el satisfactor o producto educativo, mejorar la comunicación, potenciar las relaciones públicas, conocer los productos de la competencia y conocer el mercado.

\section{b) Testimonios de egresados}

De igual manera, en este tipo de eventos se pueden emplear estrategias como presentar las experiencias de egresados que a partir de sus estudios de cuarto o quinto nivel han alcanzado significativos logros como consecuencia de sus estudios de postgrado. En contraposición con esta propuesta, está la realidad de que apenas un $18 \%$ de los consultados, manifiesta usar el testimonio de los egresados exitosos, para promocionar sus Programas de Postgrado. 


\section{c) Presentación de opciones de estudio}

También un porcentaje bastante bajo, $41 \%$, revela que hace uso de las presentaciones de opciones de estudio a los profesionales interesados en participar en los Programas de Postgrado de su Universidad.

\section{d) Publicidad}

Haciendo referencia a Russell et al. (2006) la publicidad es la rama de las comunicaciones del proceso de mercadotecnia y forma parte de la cultura cotidiana de la mayoría de los seres humanos; ésta permite crear conciencia y curiosidad por el producto para fomentar la adquisición del mismo. Para transmitirla se puede hacer uso de los medios de comunicación, desde los más tradicionales, hasta los más contemporáneos.

Con respecto al uso de los canales impersonales para la publicidad de los Programas de Postgrado, el mayor porcentaje $96 \%$, corresponde al empleo de Trípticos o Folletos; entre las ventajas de su uso están el bajo costo, la flexibilidad, y la selectividad geográfica. Un $86 \%$ de Ios encuestados utiliza la prensa, esto tiene su explicación en los beneficios que da, tales como: la cobertura y la selectividad geográfica, además el costo por persona cubierta es relativamente bajo y es de gran flexibilidad en cuanto a su formato (O'Guinn et al., 1998).

Por otro lado, un $73 \%$ utiliza Internet; considerado en la actualidad como uno de los medios de mayor alcance durante las 24 horas del día y de cobertura tanto local, regional, nacional e internacional; además ha provocado cambios significativos en lo que a medios de comunicación se refiere. Con respecto a ello, O'Guinn et al. (1998), consideran a Internet como un medio emergente, que ofrece a los negocios, posibilidades para satisfacer las necesidades de los clientes.

El empleo de la radio representa un $55 \%$; y su uso se puede fundamentar en la utilización masiva del medio, la selectividad cualitativa y geográfica, bajo costo y flexibilidad; generalmente suele emplearse como complemento de otros medios utilizados en campañas publicitarias; a esto agrega Stanton et al. (2004), este medio sólo causa una impresión auditiva, basándose enteramente en la capacidad del radioescucha para retener la información oída y no vista. Continua acotando éste que la atención de la audiencia a menudo presenta un bajo nivel, porque la radio suele emplearse como un estímulo de fondo para trabajar, estudiar o realizar alguna otra actividad.

Por último, las revistas especializadas se suelen utilizar para llegar a segmentos más específicos (Valdés et al., 2000), pero según los resultados derivados del instrumento de recolección de datos aplicado, se detectó que apenas un $23 \%$, emplea las Revistas especializadas como instrumento para promover los Programas de Postgrado, el bajo porcentaje puede atribuirse a que generalmente el tiraje de las revistas está limitado, y el mercado al cual está dirigido, habitualmente es muy específico.

Cabe destacar que apenas un $41 \%$ revela el uso frecuente de materiales promocionales para la promoción de sus Programas de Postgrado y un $100 \%$ de los encuestados no mantiene avisos permanentes en los medios de comunicación, situación que conlleva a pensar que quizás el esfuerzo hecho en la promoción 
de los Programas de Postgrado no sea del todo eficiente y no se obtenga el objetivo deseado, el cual debe ser llegar a transmitir el mensaje a la mayor cantidad de público objetivo posible.

Por otro lado, un $59 \%$ de los encargados de dirigir o coordinar las actividades promoción de los Programas de Postgrado, se sienten satisfechos en cuanto a los resultados que han obtenido de sus programas de promoción. De igual manera, las tendencias sociales y económicas, junto con los avances tecnológicos son los principales determinantes de la forma en la que se practica la publicidad en cualquier sociedad ( $O^{\prime}$ Guinn et al., 1998). Así pues, la selección del canal adecuado (personal o impersonal) va a depender de la estrategia seleccionada por el tomador de decisiones. De esta manera el uso de los canales impersonales para la transmisión de información, dirigida a un público objetivo, particularmente, los interesados en cursar estudios de cuarto y quinto nivel, constituyen un instrumento enfocado a estimular la demanda o cambiar el comportamiento del mercado meta.

Por último, cabe destacar que para completar el proceso mercadológico no sólo basta con diseñar un excelente servicio o satisfactor y colocarlo a disposición del mercado, las universidades además deben hacer esfuerzos para comunicarse con sus clientes y asegurarse de trasmitirles el mejor mensaje, utilizando los canales adecuados.

\section{Consideraciones finales}

Tal como ocurre en la mayoría de las áreas de marketing, el público objetivo o auditorio meta, influirá en las decisiones sobre la mezcla promocional, debido a que serán los clientes finales los que tendrán en si la decisión de la compra. Así pues, el objetivo en sí de la promoción es la persuasión. Si las universidades no promocionan sus productos educativos 0 satisfactores, se pierde la posibilidad de que el mercado objetivo recuerde la disponibilidad de éstos, y pueda buscar otras opciones, que les permitan satisfacer sus necesidades de formación.

La caracterización del target group al cual van dirigidos los esfuerzos de promoción, en función del perfil establecido por los diferentes Programas de Postgrado de las Universidades públicas del Estado Falcón, permitió detectar la necesidad de proporcionar mayor información sobre: Tipo de Programa ofertado, Título profesional solicitado, si trabaja en el área o no, Componente Personal, Habilidades y/o Destrezas, y Líneas de Investigación; aspectos que agregarian valor a la información que requiere el aspirante a cursar estudios de cuarto y quinto nivel.

Es posible analizar y diseñar la mezcla de promoción del mercadeo educativo a través de la Venta personal, Publicidad, Promoción y Relaciones Públicas. Sin duda alguna, las universidades deben aprovechar su valor de marca, es decir el prestigio o reputación que ostentan, ya que la misma está caracterizada implícitamente por su capacidad de agregar valor a un producto o satisfactor que ofrece. De esta manera, la marca representa el mayor valor institucional que los postgrados deben aprovechar, al momento de promocionar sus productos educativos. 
De acuerdo al estudio realizado, entre los diferentes tipos de canales personales e impersonales que se emplean en la mezcla de promoción, para lograr distintos objetivos de ventas o reconocimiento de marca, están los que ofrece la publicidad (trípticos, folletos, avisos en prensa, radio, revistas especializadas, Internet, entre otros) aun cuando el total de las unidades informantes manifiestan no mantener avisos permanentes en esos medios, algunos de ellos tampoco aprovechan los eventos académicos para promover los Programas de Postgrado, y muy pocos revelan usar el testimonio de egresados exitosos en las actividades de promoción. De igual manera, en menor grado, se revela el uso de presentaciones de opciones de estudio, a los profesionales interesados en participar en los Programas de Postgrado.

En cuanto al nivel de satisfacción de los encargados de dirigir y coordinar las actividades de promoción de los estudios de cuarto y quinto nivel, los resultados arrojaron que las unidades informantes se consideran medianamente satisfechos en cuanto a los resultados de la actividad de promoción. De esta manera, la mezcla de promoción a utilizar para comunicar al mercado meta la existencia del producto (por ejemplo, la publicidad, la venta personal, la promoción de ventas, las relaciones públicas), va a depender de las características del mercado, sus necesidades y el tipo de estrategia que se quiera emplear, además de los objetivos que se proponga cada institución.

\section{Referencias bibliográficas}

Cobra, Marcos (2001). Marketing de Servicios. Segunda Edición. Editorial McGraw Hill Interamericana.

Coughlan, Anne; Anderson, Erin; Laoudon, John; Stern, Louis; Gray, John (2006). Marketing Channels. Seventy Edition. Prentice Hall.

García, Juan (2007). Marketing Internacional. Segunda edición. Mc Graw Hill.

Hurtado De Barrera, Jacqueline (2000). Metodología de la Investigación Holística. Tercera Edición. Editorial Sypal.

Kotler, Philip (2000). Dirección de Mercadotecnia. Prentice Hall.

O'Guinn, Tomas; Allen, Chris; Semenick, Richard (1998). Publicidad. Thomson Editores.

Rodríguez, Z.; García, Y. (2002). Conceptos sobre Marketing estratégico en la actividad académica de la educación superior. Universidad de Cienfuegos. Cuba. Disponible en: http:// www.ucf.edu.cu/publicaciones/anuario2002/economicas/ articulo38.pdf. Consultado: 12-05-07.

Russell, Thomas; Lane, Ronald; Whitehill, Karen (2006). Kleppner's Advertising. Prentice Hall.

Schoell, William; Guiltinan, Joseph (1991). Mercadotecnia. Conceptos y Prácticas Modernas. Tercera Edición.

Stanton, William; Etzel Michael; Walker, Bruce (2004). Fundamentos de Marketing. $13^{\text {ava }}$ Edición. Editorial McGraw Hill.

Stern, I.; El-Ansary, A. (1988). Canales de Mercadotecnia. Prentice Hall. Enlgwood. Cliff. New Jersey.

Valdés, Joseph; Pardo, Virtudes (2000). Marketing Internacional. Ciclo formativo. Grado Superior. McGrawHill. 